\title{
Inversion Techniques for Trajectory Control of Flexible Robot Arms*
}

\author{
A. De Luca, P. Lucibello, and G. Ulivi \\ Dipartimento di Informatica e Sistemistica, \\ Università di Roma 'La Sapienza', \\ Via Eudossiana 18, 00184 Roma, Italy \\ Received January 14, 1989; accepted March 13, 1989
}

\begin{abstract}
A general framework is given for computing the torques that are needed for moving a flexible arm exactly along a given trajectory. This torque computation requires a dynamic generator system, as opposed to the rigid case, and can be accomplished both in an open- or in a closed-loop fashion. In the open-loop case, the dynamic generator is the full or reduced order inverse system associated to the arm dynamics and outputs. In order to successfully invert the arm dynamics, the torque generator should be a stable system. The stability properties depend on the chosen system output, that is on the robot variables (e.g., joint or end-effector) to be controlled. The same inversion technique can be applied for closed-loop trajectory control of flexible robots. A simple but meaningful nonlinear dynamic model of a one-link flexible arm is used to illustrate different feasible control strategies. Simulation results are reported that display the effects of the system output choice on the closed-loop stability and on the overall tracking performance.
\end{abstract}

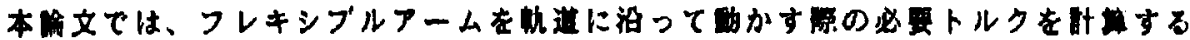

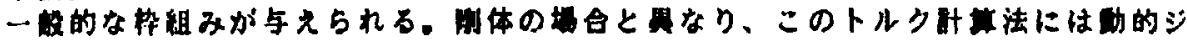

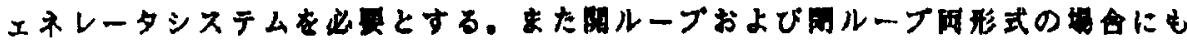

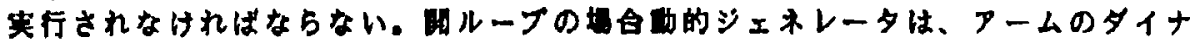

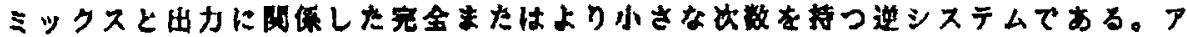

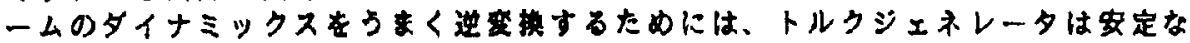
システムでなければならない。安定特性は造招されたシステム出力に传存する。そ

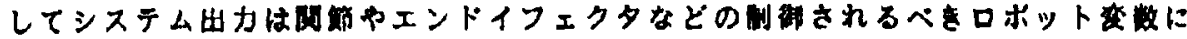

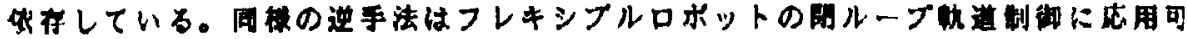

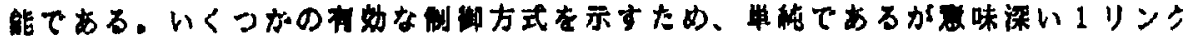

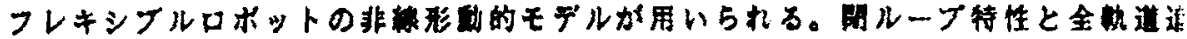

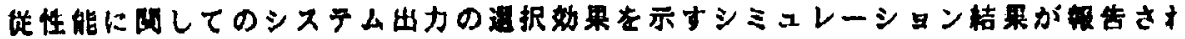
万.

*The original version of this article was presented at the Second International Symposium on Robotics and Manufacturing (ISRAM), Albuquerque, New Mexico, November 16-18, 1988. The published proceedings of this meeting may be ordered from: CAD Laboratory for Systems/Robotics, EECE Dept., UNM, Albuquerque, NM 87131. 


\section{INTRODUCTION}

Control of flexible robot arms is a challenging problem which has recently received increasing attention: Using different linear or nonlinear dynamic models, ${ }^{1-3}$ several strategies have been proposed based on linear quadratic theory, ${ }^{4}$ singular perturbations, ${ }^{5}$ adaptive control ${ }^{6.7}$ pseudo-linearization, ${ }^{8}$ and nonlinear decoupling techniques. ${ }^{9}$ Both the tracking and the point-to-point control have been considered.

It is appealing to try to find the analogue for flexible arms of the so-called computed torque or inverse control method for rigid robots. ${ }^{10}$ For rigid arms a model-based nonlinear static state-feedback transforms the closed-loop system into a linear and decoupled system made of input-output strings of double integrators. The tracking of desired trajectories is then easily achieved on the linear side of the problem. Similar results are obtained both for joint-based and for cartesian-based control schemes. ${ }^{11}$

While the extension of these nonlinear techniques to joint level control of flexible arms seems to be quite straightforward, ${ }^{9,12}$ problems arise for the end-effector trajectory control. The basic limitation is due to the noncolocation of actuators and controlled outputs. ${ }^{4.7}$ Even when considering approximated linear models for flexible arms, the nonminimum phase nature of the end-effector control problem makes the exact reproduction of trajectories a hard task.

It is still not clear how to compute torques which will move the end-effector along a desired trajectory, at least in nominal conditions. Some results were given in Ref. 13 for a linear dynamic model of a one-link flexible arm. The computation of the torque in this case is intrinsically an open-loop off-line process since it requires the a priori knowledge of the whole desired trajectory. On the other hand, instability may be a limitation of nonlinear decoupling laws for end-effector control of multilink fiexible arms. ${ }^{14}$

In this article fundamental issues related to the control problem of flexible arms are investigated using nonlinear inversion techniques ${ }^{15.16}$ as the main theoretical tool. It will be shown how the feasibility of both open-loop and closed-loop control approaches for trajectory tracking is essentially related to the stability properties of a certain dynamic system associated to the plant, the so-called reduced order inverse system ${ }^{17}$ of the input-state-output flexible arm dynamics.

With the aid of a simple nonlinear model of a one-link flexible arm, the key concepts of system inversion are illustrated and act as a guide for more complex cases. It will be shown how the selection of the output used for inversion control effects both the accuracy of the arm end-point tracking and the actuator torque requirements. Moreover, the present analysis suggests a mechanical design of the arm which yields always exact reproduction of end-effector trajectories by enforcing the stability of the inversion based controller. 


\section{ROBOT DYNAMIC MODELS}

Using a Lagrangian approach, the dynamic model of a robot arm can always be set into the standard form

$$
B(\mathbf{q}) \ddot{\mathbf{q}}+c(\mathbf{q}, \dot{\mathbf{q}})+e(\mathbf{q})=G(\mathbf{q}) \mathbf{u}
$$

where $\mathbf{q} \in R^{n}$ are the generalized coordinates of the robot system and $\mathbf{u} \in R^{m}$ are the generalized external forces acting on the system.

For rigid arms the above model is readily obtained after computation of the total kinetic and potential energy. ${ }^{10}$ In this case $n=m=N$, the number of arm joints. The same procedure applies when lumped elasticity is considered; in particular, arms with concentrated joint elasticity are modeled by similar equations but with $n=2 N, m=N .^{18}$

For distributed link flexibility, finite order approximate models can be obtained in several ways, e.g., using finite-element methods ${ }^{1}$ or assumed modes of deformation. ${ }^{2,3}$ For a flexible robot arm with $N$ actuated joints, $m=N$ and $n-m>0$ is the number of generalized coordinates used to describe flexibility.

In any case, the matrix $B$ of generalized inertia will be a positive definite and symmetric matrix for all $\mathbf{q}$, while the vectors $c$ and $e$ will respectively contain the Coriolis and centrifugal terms, and the gravitational and elastic forces. $G$ is a $n \times m$ matrix of full column rank $m$, defining the way inputs $\mathbf{u}$ act on the generalized coordinates $q$.

For ease of notation, the Einstein summation convention will be used from now on. The second order dynamics of the arm is then rewritten as

$$
\ddot{q}_{i}=d_{j i}(\mathbf{q})\left[g_{i k}(\mathbf{q}) u_{k}-c_{i}(\mathbf{q}, \dot{\mathbf{q}})-e_{i}(\mathbf{q})\right]
$$

where $d_{j i}=d_{i j}$ are elements of the inverse $D(\mathbf{q})=B^{-1}(\mathbf{q})$. The terms $c_{i}$ can be computed directly from the inertia matrix as

$$
c_{i}(\mathbf{q}, \dot{\mathbf{q}})=b_{i j, k}(\mathbf{q}) q_{j} \dot{q}_{k}-\frac{1}{2} b_{h k, i}(\mathbf{q}) \dot{q}_{h} \dot{q}_{k}
$$

where the index after the comma stands for derivative with respect to that component of $\mathbf{q}$.

Dissipative terms can be included in the model and added to the expression of $c_{i}$. In summary, noninertial, potential and dissipative terms can be grouped into a vector $n\left(\mathbf{q}, \mathbf{q}^{\prime}\right)$ of components

$$
n_{i}(\mathbf{q}, \dot{\mathbf{q}})=c_{i}(\mathbf{q}, \dot{\mathbf{q}})+e_{i}(\mathbf{q})+f_{i j} \dot{q}_{j}
$$

where the last term models friction at the motor axis and/or internal damping of the flexible structure. 


\section{INVERSION TECHNIQUES}

A set of independent output functions defining the objectives of control can be associated to the robotic system. For simplicity, outputs are chosen to depend only on the generalized coordinates $q$ (and not on $\dot{q}$ ) as

$$
\mathbf{y}=h(\mathbf{q})
$$

with $y \in R^{m}$, and the number of outputs is taken equal to the number of available control inputs. For a flexible robot, $y$ may specify any location along the arm. Accordingly, $h(q)$ will be the direct kinematics associated to this point. If a joint-level strategy is chosen, $h$ will depend only from joint coordinates, i.e., from a subset of $q$. In the following it is also assumed that the whole state $(\mathbf{q}, \dot{\mathbf{q}})$ of the system is accessible, although this is not always necessary.

Following Refs. 15 and 16 the inversion algorithm yields

$$
\begin{aligned}
y_{k} & =h_{k}(\mathbf{q}), \quad \dot{y}_{k}=h_{k, j}(\mathbf{q}) \dot{q}_{j} \\
\ddot{y}_{k} & =h_{k, j}(\mathbf{q}) \ddot{q}_{j}+h_{k, i j}(\mathbf{q}) \dot{q}_{i} \dot{q}_{j} \\
& =h_{k, j}(\mathbf{q}) d_{i j}(\mathbf{q})\left[g_{i k}(\mathbf{q}) u_{k}-n_{i}(\mathbf{q}, \dot{\mathbf{q}})\right]+h_{k, i j}(\mathbf{q}) \dot{q}_{i} \dot{q}_{j}
\end{aligned}
$$

In order to recover the inputs $u$ from the knowledge of the first two time derivatives of the outputs, the (generally not symmetric) matrix

$$
A(\mathbf{q})=J(\mathbf{q}) D(\mathbf{q}) G(\mathbf{q}), \quad J(\mathbf{q})=\frac{\partial h(\mathbf{q})}{\partial \mathbf{q}}
$$

has to be nonsingular. If all the rows of this matrix are not identically zero then $A(q)$ is also the decoupling matrix of the system. ${ }^{14}$

For rigid arms, $A(q)$ is globally invertible in the case of joint-based control strategies. For end-effector control this result holds except for singular points, where the Jacobian $J(q)$ looses full row rank. It is interesting to mention that there are robotic structures, such as planar rigid arms with concentrated elasticity at the joints, for which the above decoupling matrix is singular for all configurations $q{ }^{18}$ In these cases the inversion algorithm proceeds involving higher order output derivatives.

For robots with flexible links, the invertibility of $A(q)$ is a generic property and may fail only for special definitions of the output parameters. This concept will be illustrated further in an example in a later section. Without burdening the development, it will be assumed here that $A(q)$ has a global inverse $A^{-1}(q)$. If this were not the case, similar results could be obtained exploiting the inversion technique as in Ref. 18. Thus, the above relationships can be solved for the inputs $u$ as

$$
\begin{aligned}
u_{h} & =a_{h k}^{-1}(\mathbf{q})\left[\ddot{y}_{k}+h_{k, j}(\mathbf{q}) d_{i j}(\mathbf{q}) n_{i}(\mathbf{q}, \dot{\mathbf{q}})-h_{k, j j}(\mathbf{q}) \dot{q}_{i} \dot{q}_{i}\right] \\
& =u_{h}^{*}(\mathbf{q}, \dot{\mathbf{q}}, \ddot{\mathbf{y}})
\end{aligned}
$$


These equations will be used next to study open-loop and closed-loop control strategies.

\section{CONTROL OF FLEXIBLE ARMS}

\section{Open-Loop Control}

First, a known result for rigid manipulators is restated in the present setting. For a $\mathrm{N}$-jointed nonredundant rigid robot, the open-loop torques u that drive the system outputs $\mathbf{y}$ so as to reproduce a desired $C^{1}$-trajectory $y_{\text {des }}(t)$ are directly computed from

$$
u_{h}(t)=u_{h}^{*}\left(\mathbf{q}_{\text {des }}(t), \dot{\mathbf{q}}_{\text {des }}(t), \ddot{\mathbf{y}}_{\text {des }}(t)\right)
$$

where

$$
\mathbf{q}_{\text {des }}(t)=h^{-1}\left(\mathbf{y}_{\text {des }}(t)\right), \quad \dot{\mathbf{q}}_{\text {des }}(t)=J^{-1}\left(\mathbf{q}_{\text {des }}(t)\right) \dot{\mathbf{y}}_{\text {des }}(t)
$$

all vectors being $N$-dimensional $(n=m=N)$. This is exactly the off-line "computed torque" method for rigid robots. ${ }^{10,11}$ To fix ideas, the above pair of relations may be the standard inverse of the manipulator direct and differential kinematics. Due to the presence of the second time-derivative of the output in the inversion process, exact output reproduction of a desired trajectory is possible only if this has bounded acceleration. Moreover, the system initial conditions (i.e., $q(0)$ and $\dot{q}(0)$ ) have to be matched with the desired ones. ${ }^{12}$

A more involved situation arises in flexible robots, where $n>m$. For this class of arms, the knowledge of $\mathbf{y}_{\text {des }}(t)$ and of its time derivatives is not enough to determine the required torques instantaneously, i.e., in a static way. Instead, a dynamic inverse system has to be used inside the open-loop torque generator. The physical purpose of this additional dynamics is to generate the natural behavior of those system variables which are not directly constrained by the outputs specification. Such a natural behavior is the one obtained under the action of the inversion-based intput $u^{*}$ in (1). This inverse dynamic system may be a full or reduced order one.

\section{Full Order Inversion}

According to the general method, ${ }^{15}$ the required open-loop torques are obtained as follows. Given $y_{\text {des }}^{\prime \prime}(t)$ and matched initial state conditions, integrate the $n$ second order nonlinear ODE

$$
\ddot{q}_{j}=d_{i j}(\mathbf{q}) g_{i k}(\mathbf{q}) u_{k}^{*}\left(\mathbf{q}, \dot{\mathbf{q}}, \ddot{y}_{\text {des }}\right)-d_{i j}(\mathbf{q}) n_{i}(\mathbf{q}, \dot{\mathbf{q}})
$$

which describe the robot full dynamics under the action of the input $\mathbf{u}^{*}$ in (1). Note that only the highest (second) order derivative of the desired output is needed here. Moreover, $\ddot{y}_{\text {des }}$ enters into the system in a linear fashion, acting as an input. 
Labelling $\mathbf{q}_{\mathrm{des}}(t)$ and $\dot{\mathbf{q}}_{\mathrm{des}}(t)$ the time evolutions obtained by integration, the torques needed to follow the desired trajectory are computed as

$$
\begin{aligned}
u_{h}(t)= & a_{h k}^{-1}\left(\mathbf{q}_{\mathrm{des}}(t)\right)\left[\ddot{y}_{k, \text { des }}(t)+h_{k, i}\left(\mathbf{q}_{\mathrm{des}}(t)\right) d_{i j}\left(\mathbf{q}_{\mathrm{des}}(t)\right) n_{i}\left(\mathbf{q}_{\mathrm{des}}(t), \dot{\mathbf{q}}_{\mathrm{des}}(t)\right)\right. \\
& \left.-\boldsymbol{h}_{k, i j}\left(\mathbf{q}_{\mathrm{des}}(t)\right) \dot{q}_{i, \mathrm{des}}(t) \dot{\boldsymbol{q}}_{j, \mathrm{des}}(t)\right]
\end{aligned}
$$

which is the torque reference produced by the dynamic generator that will be fed into the flexible manipulator. Indeed, if integration is performed with infinite precision then $\mathbf{q}_{\mathrm{des}}(t)$ and $\dot{\mathbf{q}}_{\mathrm{des}}(t)$ satisfy

$$
h\left(\mathbf{q}_{\text {des }}(t)\right)=\mathbf{y}_{\text {des }}(t), \quad J\left(\mathbf{q}_{\text {des }}(t)\right) \dot{q}_{\text {des }}(t)=\dot{\mathbf{y}}_{\text {des }}(t)
$$

\section{Reduced Order Inversion}

Full order inversion requires the integration of $n$ second-order differential equations. However, from the inversion algorithm $m$ independent algebraic equations are obtained which may be used to determine $m$ generalized coordinates. The remaining $n-m$ coordinates are generated without redundancy via a reduced inverse dynamic system of order $n-m$.

First, partition $q$ into $\left(q^{a}, q^{b}\right)$ with $q^{a} \in R^{n-m}, q^{b} \in R^{m}$ in such a way that $q^{b}$ is uniquely determined from $\mathbf{y}_{\text {des }}(t)$ and $q^{a}(t)$ as the $q_{\text {des }}^{b}\left(y_{\text {des }}, q^{a}\right)$ which satisfies

$$
h\left(\mathbf{q}^{a}, \mathbf{q}_{\text {des }}^{b}\right)=\mathbf{y}_{\text {des }}
$$

Note that not any partition of $q$ is valid. The implicit function theorem has to be invoked for the explicitability of the chosen $q^{b}$. This implies that in the equation

$$
J\left(\mathbf{q}^{a}, \mathbf{q}_{\text {des }}^{b}\right)\left[\begin{array}{c}
\dot{\mathbf{q}}^{a} \\
\dot{\mathbf{q}}_{\text {des }}^{b}
\end{array}\right]=\dot{\mathbf{y}}_{\text {des }}
$$

the corresponding partition of the Jacobian $J$ into two blocks $\left[J^{a}, J^{b}\right]$ is such that locally $J^{b}$ is nonsingular. Consequently $\dot{\mathbf{q}}_{\mathrm{des}}^{b}\left(\mathbf{y}_{\mathrm{des}}, \dot{\mathbf{y}}_{\mathrm{des}}, \mathbf{q}^{a}, \dot{\mathbf{q}}^{a}\right)$ is computed also from (4).

Hence, for a given $\ddot{y}_{\text {des }}(t)$ and matched initial state conditions, the integration of the following $n-m$ second order nonlinear ODE

$$
\begin{aligned}
\ddot{q}_{J}^{a}= & d_{i j}\left(\mathbf{q}^{a}, \mathbf{q}_{\text {des }}^{b}\right) \mathbf{g}_{i k}\left(\mathbf{q}^{a}, \mathbf{q}_{\text {des }}^{b}\right) \mathbf{u}_{k}^{*}\left(\mathbf{q}^{a}, \mathbf{q}_{\text {des }}^{b}, \dot{\mathbf{q}}^{a}, \dot{\mathbf{q}}_{\text {des }}^{b}, \ddot{\mathbf{y}}_{\text {des }}\right) \\
& -d_{i j}\left(\mathbf{q}^{a}, \mathbf{q}_{\text {des }}^{b}\right) n_{i}\left(\mathbf{q}^{a}, \mathbf{q}_{\text {des, }}^{b}, \dot{\mathbf{q}}^{a}, \dot{\mathbf{q}}_{\text {des }}^{b}\right)
\end{aligned}
$$

gives the associated behavior for $\mathbf{q}_{\text {dess }}^{a}(t)$ and $\dot{\mathbf{q}}_{\text {des }}^{a}(t)$. With the obtained timeprofiles, the open-loop torques are determined as before using (3).

It is worth noting that in the reduced order inverse dynamic equations, beside the desired output acceleration, also the desired output and its first time-derivative appear through $\mathbf{q}_{\text {des }}^{b}$ and $\dot{\mathbf{q}}_{\text {des. }}^{b}$. 


\section{Closed-Loop Control}

The above analysis was performed having in mind the open-loop determination of torques capable of moving the chosen outputs of the robotic system exactly along a given trajectory. When the system is in its nominal conditions, these computed torques force the robot to behave as desired. As usual, feeding back the currerit state may improve disturbance rejection and counterbalance perturbations due to off-nominal conditions. This is obtained at the cost of additional measurement and real-time processing capabilities.

The closed-loop strategy is derived directly from the previous inversion algorithm and provides the following nonlinear static state-feedback law:

$$
\begin{aligned}
\mathbf{u} & =[J(\mathbf{q}) D(\mathbf{q}) G(\mathbf{q})]^{-1}[\mathbf{v}+J(\mathbf{q}) D(\mathbf{q}) n(\mathbf{q}, \dot{\mathbf{q}})-\dot{J}(\mathbf{q}, \dot{\mathbf{q}}) \dot{\mathbf{q}}] \\
& =\mathbf{u}^{*}(\mathbf{q}, \dot{\mathbf{q}}, \mathbf{v})
\end{aligned}
$$

where $\mathbf{v}$ is the additional control input. The closed-loop system behavior is described in a canonic form, once the new generalized coordinates $\left(q^{a}, \mathbf{y}\right)$ are used in place of $\mathbf{q}=\left(\mathbf{q}^{a}, \mathbf{q}^{b}\right)$ :

$$
\begin{aligned}
\ddot{\mathbf{y}} & =\mathbf{v} \\
\ddot{\mathbf{q}}_{j}^{a} & =d_{i j}\left(\mathbf{q}^{a}, \mathbf{y}\right)\left[g_{i k}\left(\mathbf{q}^{a}, \mathbf{y}\right) u_{k}^{*}\left(\mathbf{q}^{a}, \mathbf{y}, \dot{\mathbf{q}}^{a}, \dot{\mathbf{y}}, \mathbf{v}\right)-n_{i}\left(\mathbf{q}^{a}, \mathbf{y}, \dot{\mathbf{q}}^{a}, \dot{\mathbf{y}}\right)\right]
\end{aligned}
$$

The first set of Eq. (7) describes the input-output relation between $v$ and $y$, which is linear and decoupled. Stabilization of the input-output behavior can be realized using standard linear techniques in the design of v, e.g., by PD control. The second set of equations is the unobservable part of the system, the so-called sink. Note that a forcing term $\mathbf{v}$ appears in these equations.

\section{STABILITY ISSUES}

The three proposed control schemes are shown in Figure 1. The actual feasibility of any of these approaches relies on the stability properties of an associated dynamic system: respectively, the full order inverse dynamics, the reduced order inverse dynamics and the unobservable dynamics. Under the working hypothesis of invertibility of the decoupling matrix $A(q)$, it is possible to prove that the stability properties of these three dynamic systems are just the same. ${ }^{17}$ Stated differently, the open-loop and the closed-loop implementation of the same inversion control strategy either both work or both fail.

The study of the global stability of the unobservable dynamics is not an easy task. The presence of the reference input $v$ makes it a nonstationary system, which in the general case is a nonlinear one. Indeed, the stability of the local linear approximation may be studied. However, interesting results are obtained by considering the stability properties of the zero-dynamics associated to the robotic system. In general, this is the dynamics which is left in a given nonlinear system once the input is chosen in such a way as to constrain the 

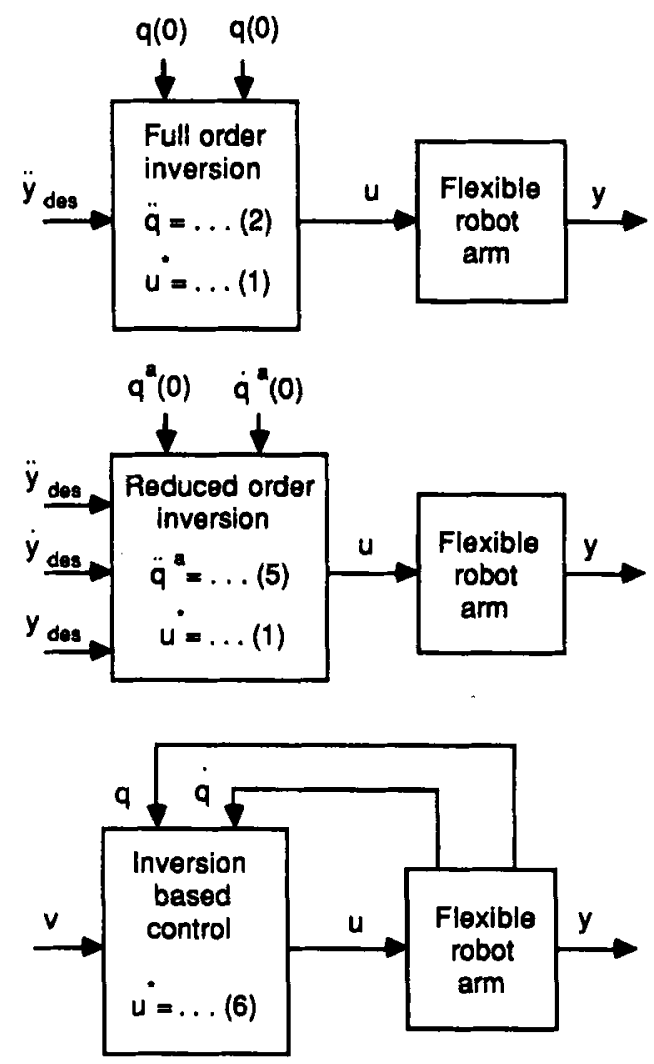

Figure 1. Open-loop and closed-loop control schemes.

output to be zero (or constant). ${ }^{17}$ This concept collapses into the ordinary one of zeros of the transfer function if the given system is linear. It is proved in Ref. 19 that in order to conclude on the local stability of the overall system, it is enough to show asymptotic stability of this dynamics. In analogy with the linear case, nonlinear systems with asymptotically stable zero-dynamics are called minimum-phase systems.

For the flexible robot arm, chosen an output $y$, the zero-dynamics is obtained when $\mathbf{y}(t) \equiv 0$. From the first row of (7), this implies also $\dot{\mathbf{y}}=\mathbf{v}=\mathbf{0}$ which substituted in the second row gives the actual expression of the zero-dynamics. This dynamical behavior is strictly related to the one associated with the elastic coordinates, once the control loop has been closed. Ensuring local stability of these variables may already be a satisfactory result, due to the small deformations in play.

It is worth poting that, in spite of dynamic instability, a particular choice of initial conditions for the elastic variables $\mathbf{q}^{a}$ describing the zero-dynamics in (7), may possibly lead to a bounded evolution of these in time. As a consequence, the output trajectory may be reproduced in a stable way if the whole 
system is properly initialized at time $t=0$. This is a way of explaining also the results obtained in Ref. 13. However, these suitable initial conditions vary in dependence of the whole trajectory to be tracked and therefore the initialization of the system is necessarily an off-line non-causal procedure.

\section{A CASE STUDY}

In order to illustrate some possible strategies for the exact trajectory control of flexible robot arms, and their inherent limitations, a simple one-link planar arm will be considered. The link flexibility is modeled by one linear torsional spring located at a generic point along the link. The link is driven at the joint by a direct drive motor. Thus, $n=2$ and $m=N=1$.

The two generalized coordinates are: $q_{1}$, which denotes the rigid rotation at the motor hub, and $q_{2}$, the flexible angular rotation. The link is divided into two rigid parts (Fig. 2), each of length $l_{i}$, mass $m_{i}$, center of mass at $l_{c i}$ and inertia $I_{i}$ with respect to this point. Motor and payload mass and inertia can be included in the proximal and distal part of the link. At the motor axis, the viscous friction coefficient is $f_{1}$. The spring has an clasticity constant $k$ and a damping factor $f_{2}$, modeling the internal frictions in the structure.

The dynamic model is the following:

$$
\left[\begin{array}{cc}
b_{11}\left(q_{2}\right) & b_{12}\left(q_{2}\right) \\
b_{12}\left(q_{2}\right) & b_{22}
\end{array}\right]\left[\begin{array}{l}
\ddot{q}_{1} \\
\ddot{q}_{2}
\end{array}\right]+\left[\begin{array}{c}
c_{1}\left(q_{2}, \dot{q}_{1}, \dot{q}_{2}\right)+f_{1} \dot{q}_{1} \\
c_{2}\left(q_{2}, \dot{q}_{1}\right)+k q_{2}+f_{2} \dot{q}_{2}
\end{array}\right]=\left[\begin{array}{l}
1 \\
0
\end{array}\right] u
$$

where

$$
\begin{aligned}
& b_{11}\left(q_{2}\right)=a+2 c \cos q_{2}, \quad b_{12}\left(q_{2}\right)=b+c \cos q_{2}, \quad b_{22}=b \\
& c_{1}\left(q_{2}, \dot{q}_{1}, \dot{q}_{2}\right)=-c\left(\dot{q}_{2}^{2}+2 \dot{q}_{1} \dot{q}_{2}\right) \sin q_{2} \\
& c_{2}\left(q_{2}, \dot{q}_{1}\right)=c \dot{q}_{1}^{2} \sin q_{2}
\end{aligned}
$$

and the model coefficients have the expressions

$$
a=I_{1}+m_{1} l_{c 1}^{2}+I_{2}+m_{2}\left(l_{1}^{2}+l_{c 2}^{2}\right), \quad b=I_{2}+m_{2} l_{c 2}^{2}, \quad c=m_{2} l_{1} l_{c 2}
$$

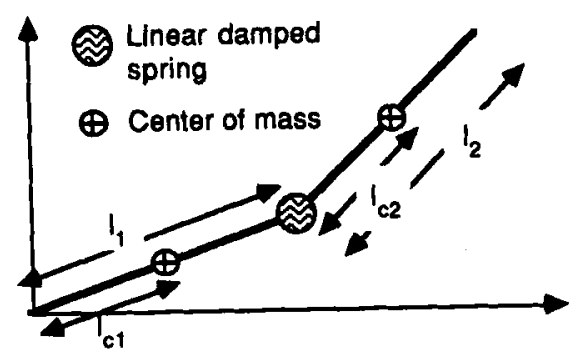

Figure 2. A simple model of flexibility for a one-link robot. 
As output of the system a parametrized function can be taken which is a linear approximation of the angle $\alpha$ to a generic point $P$ on the flexible link, as seen from the motor axis (Fig. 3). Since

$$
a=q_{1}+\arctan \frac{\lambda l_{2} \sin q_{2}}{l_{1}+\lambda l_{2} \cos q_{2}}
$$

then

$$
y=q_{1}+\frac{\lambda l_{2}}{l_{1}+\lambda l_{2}} q_{2}:=q_{1}+p(\lambda) q_{2}
$$

For $\lambda=0, y=q_{1}$ and the control strategy is performed at the joint-level; for $\lambda=1$, a task-level strategy is chosen since $y$ is now the angle pointing at the end-effector.

The inversion algorithm gives after two derivatives:

$$
\begin{aligned}
\ddot{y} & =\ddot{q}_{1}+p(\lambda) \ddot{q}_{2}=\left[\begin{array}{ll}
1 & p(\dot{\lambda})
\end{array}\right]\left[\begin{array}{ll}
d_{11} & d_{12} \\
d_{12} & d_{22}
\end{array}\right]\left[\begin{array}{c}
u-c_{1}-f_{1} \dot{q}_{1} \\
-c_{2}-k q_{2}-f_{2} \dot{q}_{2}
\end{array}\right] \\
& =\left(d_{11}+p(\lambda) d_{12}\right)\left(u-c_{1}-f_{1} \dot{q}_{1}\right)-\left(d_{12}+p(\lambda) d_{22}\right)\left(c_{2}+k q_{2}+f_{2} \dot{q}_{2}\right)
\end{aligned}
$$

where the arguments of the various functions have been dropped. The output parameter $\lambda$ has to be chosen such that

$$
p(\lambda) \neq-\frac{d_{11}}{d_{12}}=\frac{b_{22}}{b_{12}}=\frac{b}{b+c \cos q_{2}}
$$

in correspondence to all values attained by $q_{2}$. This guarantees that the output acceleration depend explicitly on the applied torque (i.e., the scalar $A(q) \neq 0$ ). Then, the above relation can be solved for the torque $u$ giving in analogy to (1):

$$
\begin{aligned}
u & =\left(c_{1}+f_{1} \dot{q}_{1}\right)+\frac{1}{d_{11}+p(\lambda) d_{12}}\left[\ddot{y}+\left(d_{12}+p(\lambda) d_{22}\right)\left(c_{2}+k q_{2}+f_{2} \dot{q}_{2}\right)\right] \\
& =u^{*}(\mathbf{q}, \dot{\mathbf{q}}, \ddot{y})
\end{aligned}
$$

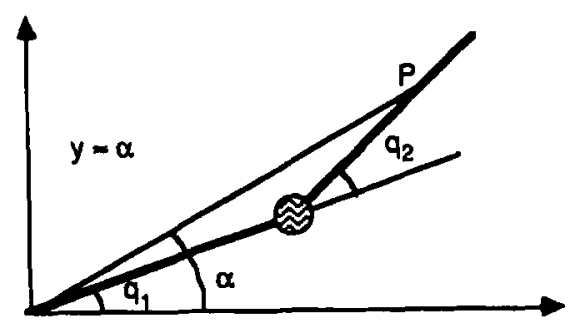

Figure 3. State and output definitions for the flexible link. 
To generate the open-loop torque required to move point $P$ with a given angular acceleration $\ddot{y}_{\text {des }}, u=u^{*}\left(\mathbf{q}, \dot{\mathbf{q}}, \ddot{y}_{\text {des }}\right)$ is plugged into the full dynamic equations of the arm as in (2) and these are integrated, starting from the current initial conditions.

To obtain a reduced order torque generator, $q$ is partitioned into $q^{a}=q_{2}$, the elastic coordinate, and $q^{b}=q_{1}$. Using for $q_{1}$ and its time-derivative the expressions

$$
q_{1}=y-p(\lambda) q_{2}, \quad \dot{q}_{1}=\dot{y}-p(\lambda) \dot{q}_{2}
$$

the reduced dynamics to be integrated is simply the dynamic equation of $q_{2}$, with $u=u^{*}\left(q_{2}, \dot{q}_{2}, y_{\text {des }}, \dot{y}_{\text {des }}, \ddot{y}_{\text {des }}\right)$ substituted therein as in (5).

As pointed out in the previous section, the stability of both these two open-loop processes is equivalent to the stability of the closed-loop system obtained setting $\ddot{y}=v$ and using (10) as a nonlinear static state-feedback law on the robot arm. In particular, the equation of the unobservable part is found from

$$
\begin{aligned}
\ddot{q}_{2} & =-\left[\begin{array}{ll}
d_{12} & d_{22}
\end{array}\right]\left[\begin{array}{c}
u^{*}-c_{1}-f_{1} \dot{q}_{1} \\
-c_{2}-k q_{2}-f_{2} \dot{q}_{2}
\end{array}\right] \\
& =\frac{d_{12}}{d_{11}+p(\lambda) d_{12}}\left[\ddot{y}+\left(d_{12}+p(\lambda) d_{22}\right)\left(c_{2}+k q_{2}+f_{2} \dot{q}_{2}\right)\right]-d_{22}\left(c_{2}+k q_{2}+f_{2} \dot{q}_{2}\right)
\end{aligned}
$$

Using the relations between the minor of a matrix and the elements of its inverse, it is possible to simplify terms. The closed-loop equations are rewritten as

$$
\begin{aligned}
\ddot{y} & =v \\
\ddot{q}_{2} & =-\frac{b_{12}\left(q_{2}\right)}{b_{22}-p(\lambda) b_{12}\left(q_{2}\right)} v-\frac{c_{2}\left(q_{2}, \dot{q}_{2}, \dot{y}\right)+k q_{2}+f_{2} \dot{q}_{2}}{b_{22}-p(\lambda) b_{12}\left(q_{2}\right)}
\end{aligned}
$$

where the new coordinates $\left(y, q_{2}\right)$ have been used. It is evident that the critically stable input-output behavior can be stabilized by pole-placement.

The associated zero-dynamics is computed by setting $y=\dot{y}=v=0$; since

$$
c_{2}\left(q_{2}, \dot{q}_{2}, 0\right)=c p^{2}(\lambda) \dot{q}_{2}^{2} \sin q_{2}
$$

the zero-dynamics is given by

$$
\ddot{q}_{2}=-\frac{c p^{2}(\lambda) \dot{q}_{2}^{2} \sin q_{2}+k q_{2}+f_{2} \dot{q}_{2}}{b-p(\lambda)\left(b+c \cos q_{2}\right)}
$$

where $p(\lambda)=\lambda l_{1} /\left(l_{1}+\lambda l_{2}\right)$. Local asymptotic stability of the equilibrium point 
$q_{2}=\dot{q}_{2}=0$ of this system is guaranteed for

$$
0 \leq \lambda<\lambda^{0} \quad \text { with } \lambda^{0}=\frac{b l_{1}}{c l_{2}}=\frac{I_{2}+m_{2} l_{c 2}^{2}}{m_{2} l_{2} l_{2}}>0
$$

Choosing the nondimensional parameter $\lambda$ in the above interval ensures that the given control scheme, beside imposing the desired time-profile to the output, leads also to a stable closed-loop behavior. By analogy, the same conclusion can be drawn for the open-loop torque generation process.

A series of remarks are now in order.

Remark 1 . The value $\lambda^{\circ}$ has a nice physical interpretation. Consider the arm at rest in its undeformed configuration. Hence, $q(0)$ is such that $q_{2}(0)=0$. At time $t=0$, apply a unitary step torque; the system accelerations are then

$$
\ddot{\mathbf{q}}(0)=\left[\begin{array}{l}
\ddot{q}_{1}(0) \\
\ddot{q}_{2}(0)
\end{array}\right]=D(\mathbf{q}(0))\left[\begin{array}{l}
1 \\
0
\end{array}\right]=\left[\begin{array}{l}
d_{11}(\mathbf{q}(0)) \\
d_{12}(\mathbf{q}(0))
\end{array}\right]=\frac{1}{\operatorname{det} B(q(0))}\left[\begin{array}{c}
b \\
-(b+c)
\end{array}\right]
$$

In correspondence to the computed $\lambda^{\circ}$ the parametrized output takes on the form

$$
y_{\lambda} \cdot(t)=q_{1}(t)+\frac{b}{b+c} q_{2}(t)
$$

Thus, $\ddot{y}_{\lambda^{\circ}}(0)=0$; the point $P\left(\lambda^{\circ}\right)$ on the link is the one with initial zero angular acceleration.

Remark 2. The same interpretation of $\lambda^{\circ}$ applies if a dynamic model of the arm is used which is linearized around the undeformed configuration. In this case an input-output transfer function can be associated to the state space model. Then, $\lambda^{\circ}$ specifies the boundary in the output definition between systems of minimum phase (having pairs of zeros on the imaginary axis) and of non-minimum phase (having positive/negative pairs of real zeros).

Remark 3. The choice $\lambda=0$ is always a feasible one and results in a joint-based control strategy. This case has already been considered in. . $^{9.12}$ Without additional control action, this approach leads to oscillations of the end-effector which are generally only lightly damped.

Remark 4. Depending on the mechanical structure, the value of $\lambda^{\circ}$ may or may not be larger than 1 . In the first case, this implies that a desired trajectory can be assigned to the end-effector in a stable way. Otherwise, instability occurs in the associated open-loop torque generation and in the relative closed-loop control strategy (see e.g., Ref. 14 for a two-link example).

Remark 5. If each sublink of the arm described by (8) is assumed to be a uniform thin rod, it is easy to see that $\lambda^{\circ}=2 / 3$. Note that all of the above considerations hold also in the case of distributed elasticity. For example, a flexible beam with one parabolic deformation mode ${ }^{3}$ and uniform mass distribution has $\lambda^{\circ}=4 / 5$, closer to the arm tip. 
Remark 6. The optimal choice of $\lambda$ in the given interval is an interesting issue. If $\lambda^{\circ}$ is strictly less than 1 , then performance can be evaluated in terms of the actual behavior of the end-effector for different admissible values of $\lambda$.

\section{SIMULATION RESULTS}

The proposed inversion control law for trajectory tracking has been simulated on the flexible one-link arm (8) using the following set of parameters: $l_{i}=0.5 \mathrm{~m}, l_{c i}=0.25 \mathrm{~m}, m_{i}=0.1 \mathrm{~kg}, f_{i}=0.01 \mathrm{Nm} \mathrm{s} / \mathrm{rad}$, for $i=1,2$. The spring elasticity is $K=10 \mathrm{Nm} / \mathrm{rad}$. The desired angular trajectory to be tracked has a bang-bang symmetric acceleration profile; its maximum value is $4 \mathrm{rad} / \mathrm{s}^{2}$ and the whole trajectory is $0.6 \mathrm{~s}$ long, resulting in a total rotation of $0.36 \mathrm{rad}$. A second-order Runge-Kutta method is used for integration with a $1 \mathrm{msec}$ step size.

A first set of simulations was performed for the case of uniform mass distribution $\left(I_{i}=m_{i} l_{i}^{2} / 12, i=1,2\right)$ and is reported in Figures 4-6. In this case $\lambda^{\circ}=2 / 3$ and the results are relative to three feasible values of $\lambda$, respectively $0,0.3$ and 0.6. The plots shown are the angular output $y(\lambda)$ in (9), the angular motion $y(1)$ of the tip, their difference $d=y(\lambda)-y(1)$, the applied torque $u$ in (10), and the evolution of the two coordinates $q_{1}$ and $q_{2}$.

In all cases the controlled output $y(\lambda)$ behaves as desired. It is easy to see that moving from a joint-based control strategy $(\lambda=0)$ towards inversion control of a point along the link implies a benefit on the accuracy of the tip trajectory, with a large reduction of its vibratory behavior. The price to pay is an increased torque requirement. Note that the torque oscillates around average values of $\pm 0.27 \mathrm{Nm}$, which are the ones that would be required if the arm was rigid. Indeed, a smoother reference trajectory used in place of the bang bang profile, would result in smaller excursions of the torque.

When a $\lambda$ larger than $\lambda^{\circ}$ is attempted for directly assigning the behavior of the arm tip, instability occurs in the inversion-based control scheme. This results in an explosion of the closed-loop torque just after few instants of simulation. The same happens in the process of open-loop torque generation.

It can be seen from the analytic expression of $\lambda^{\circ}$ that this critical value can be increased by adding inertia $I_{2}$ to the distal sublink and/or pushing further the location $l_{\mathrm{c} 2}$ of its center of mass. A redistribution of masses helps to this purpose. Note also that adding a payload to the arm has a similar positive effect. If $l_{c 2}$ is not modified, an inertia $I_{2}$ three times as large as in the uniform case would bring $\lambda^{\circ}$ to 1 , thus enabling exact control of the tip motion.

Figures 7 and 8 refers to the case when the mechanical parameters are such that $\lambda^{\circ}=1.5$. In this case, using $\lambda=1$ leads to a feasible approach and this is confirmed by the exact tracking of the tip angular trajectory shown in Figure 7 (a). In this case the internal variable $q_{2}$ vibrates in counterphase with $q_{1}$ (note the different scales in Figure $7(b)$ ). Therefore the torque is still oscillatory and the total requirement is not reduced as compared to the one in Figure 6(b). However, we may somewhat relax the end-effector tracking accuracy by choosing again $\lambda=0.6$. Figure 8 refers to this case and confirms that a 


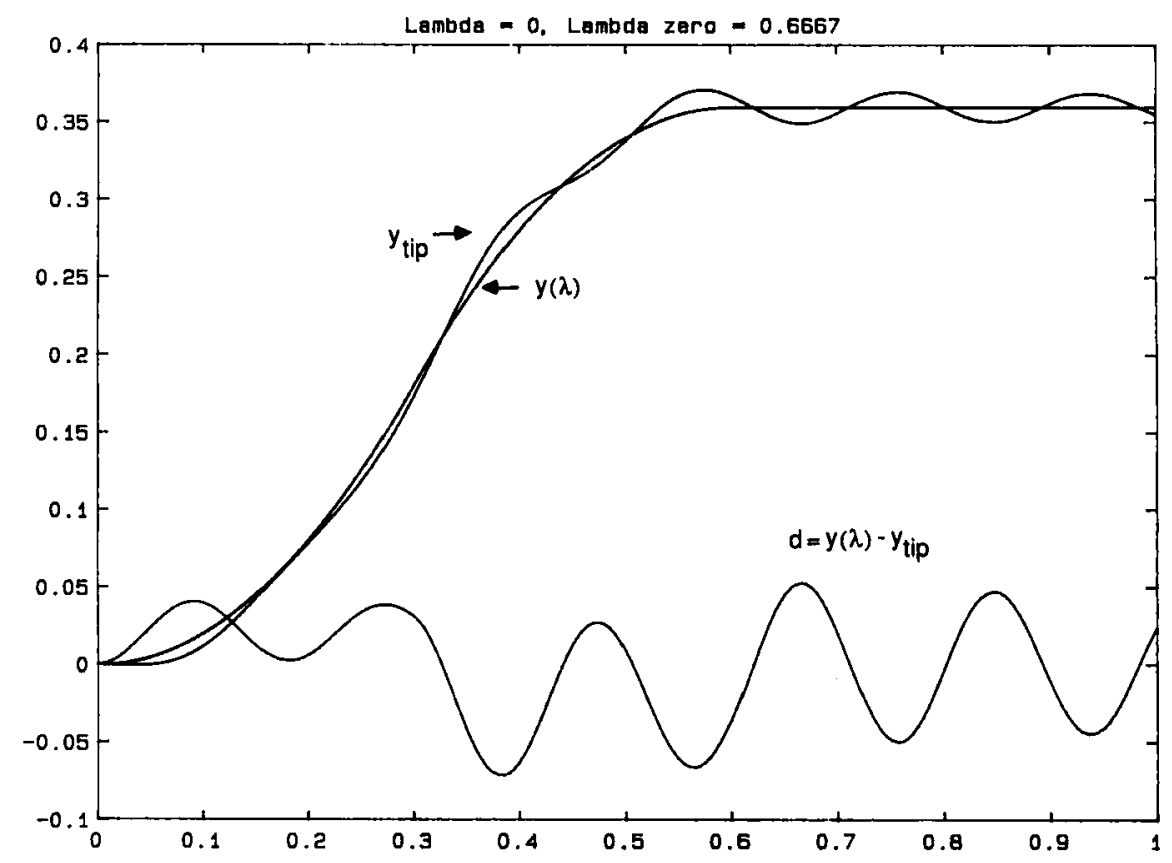

Figure 4(a). Outputs and their difference ( $d$ is multiplied by 5 ).

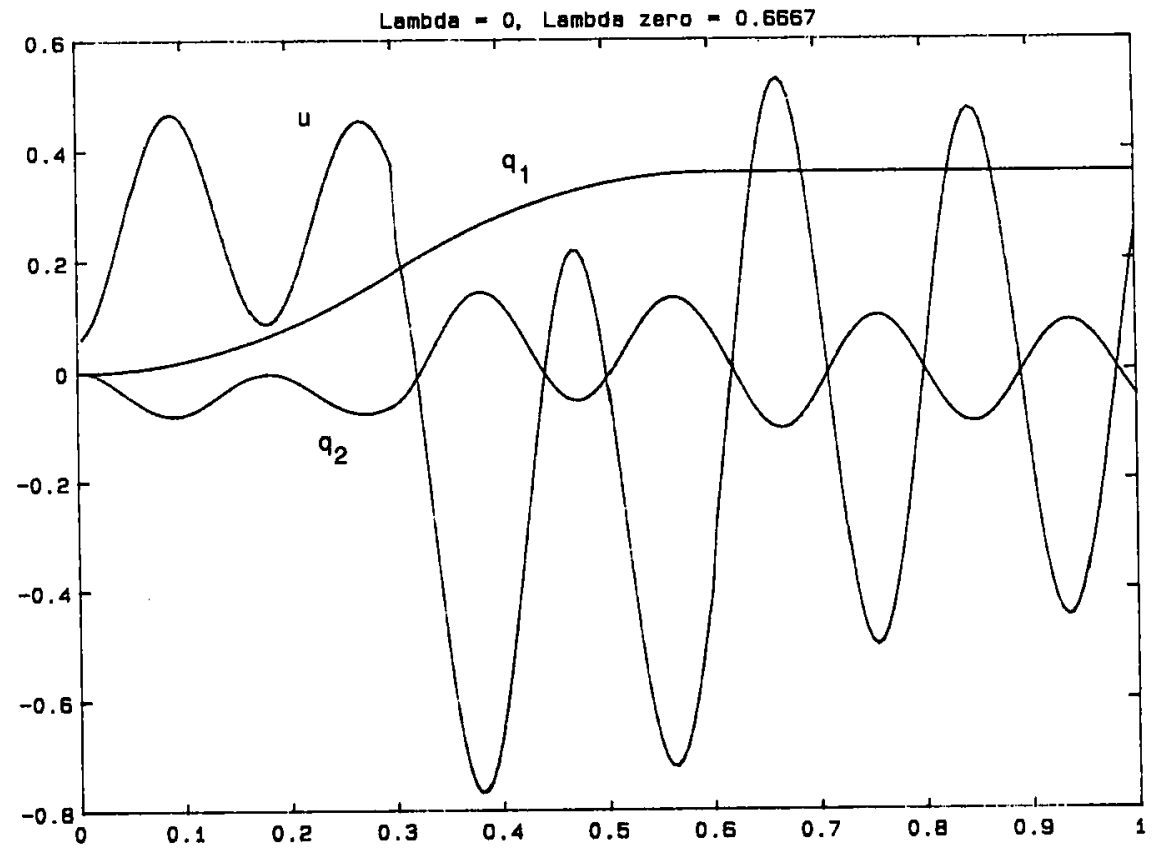

Figure 4(b). Torque, $q_{1}$ and $q_{2}\left(q_{2}\right.$ is multiplied by 5$)$. 


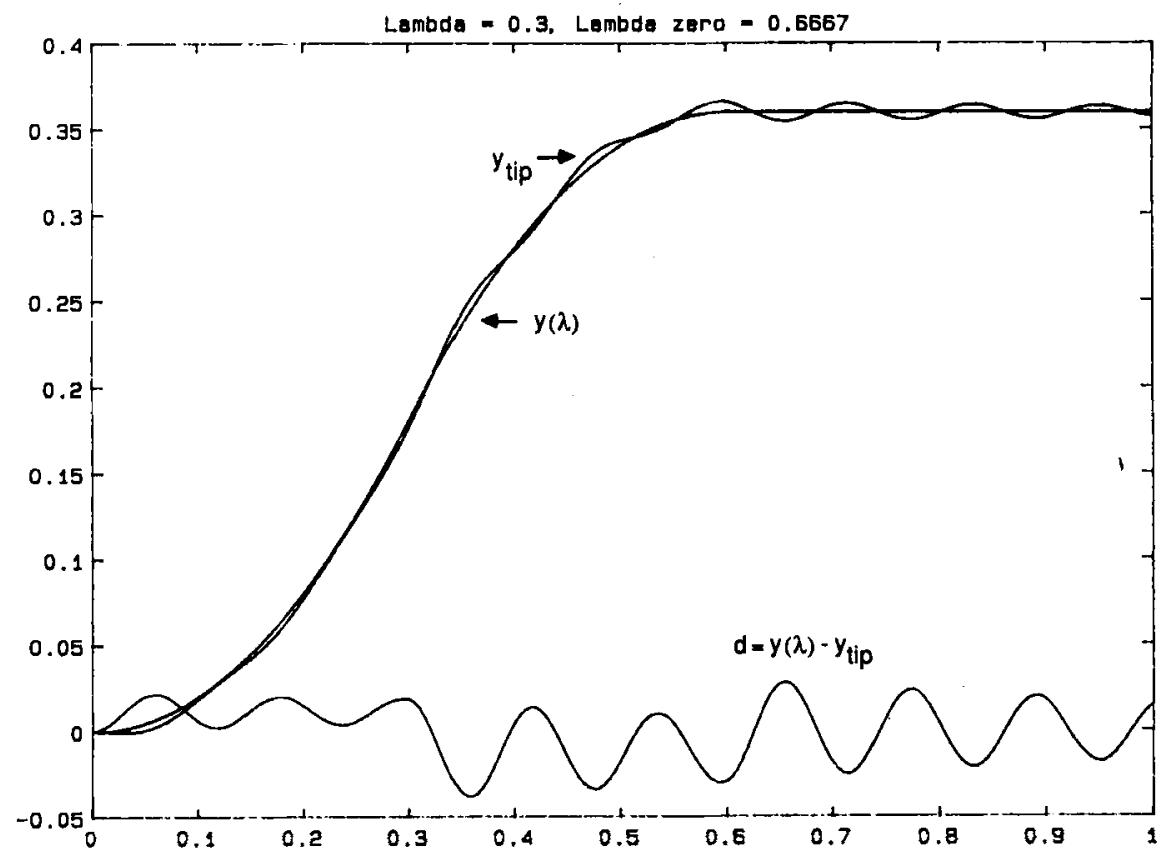

Figure 5(a). Outputs and their difference ( $d$ is multiplied by 5 ).

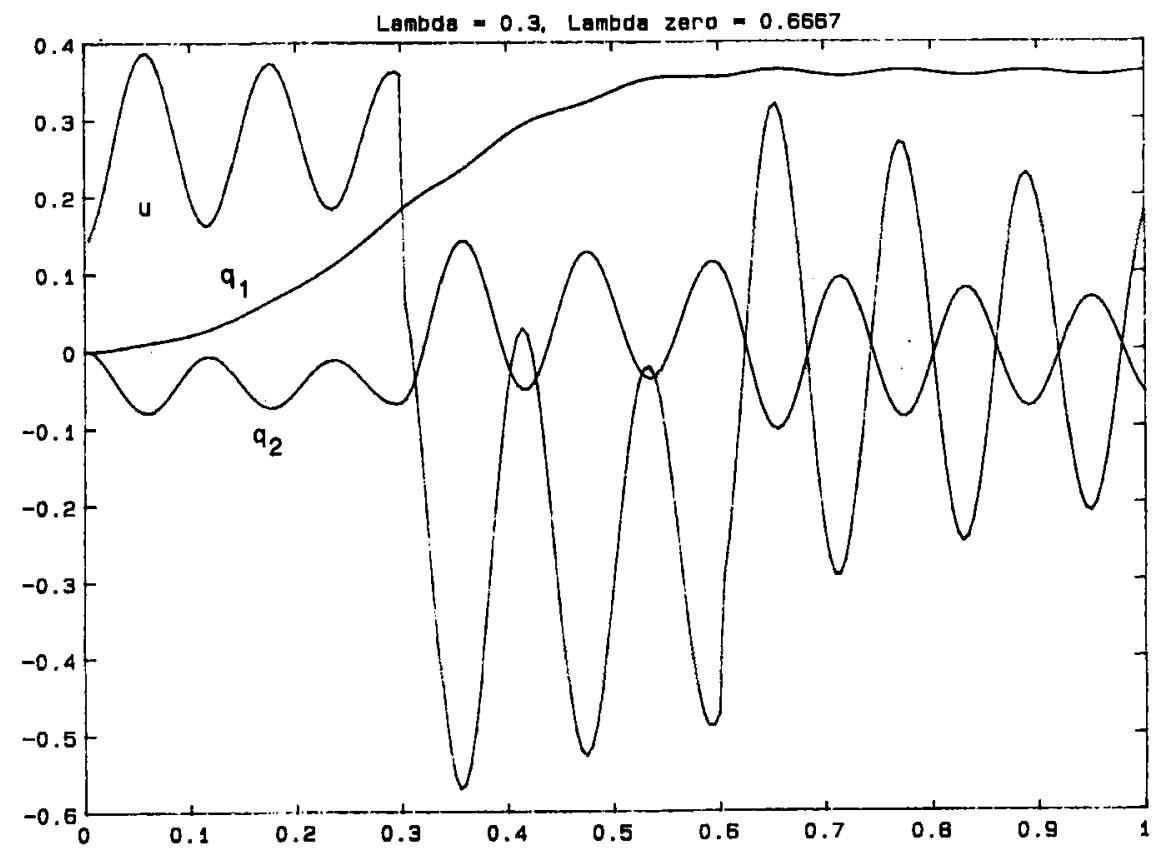

Figure 5(b). Torque, $q_{1}$ and $q_{2}$ ( $q_{2}$ is multiplied by 5 ). 


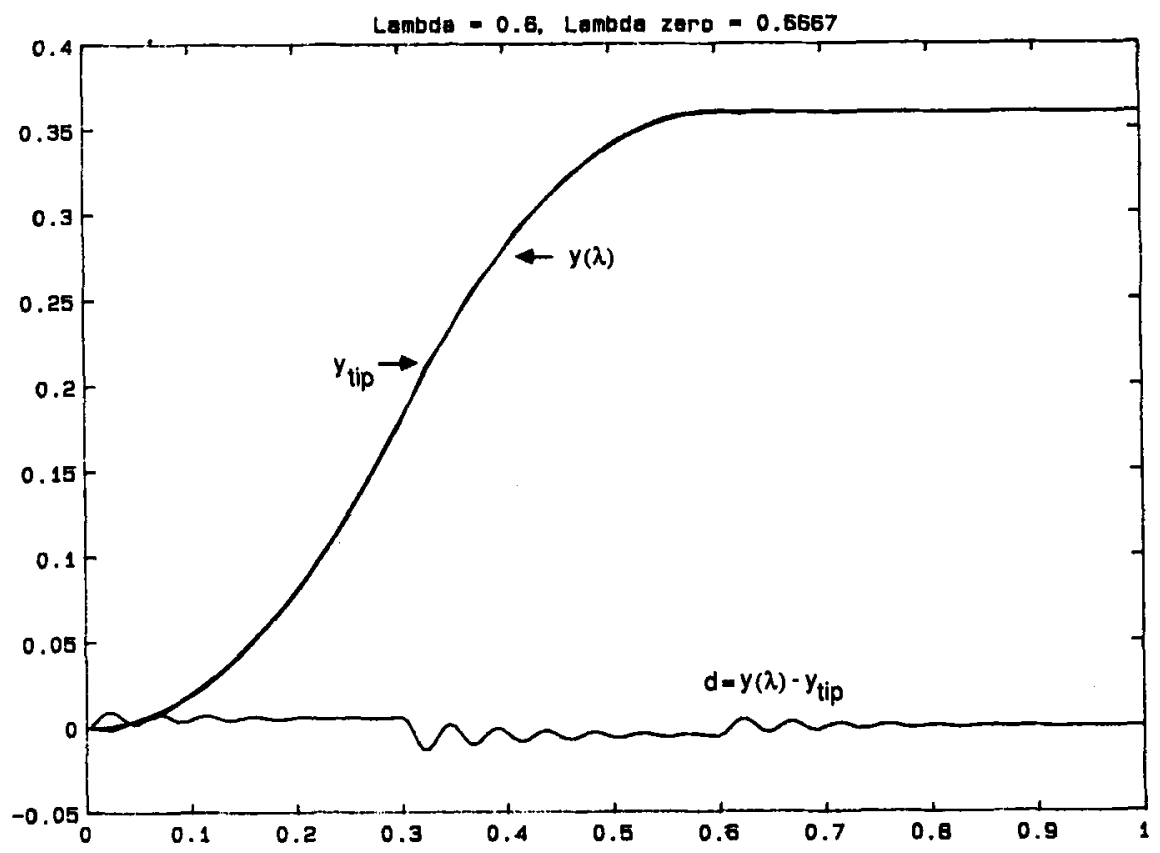

Figure 6(a). Outputs and their difference ( $d$ is multiplied by 5 ).

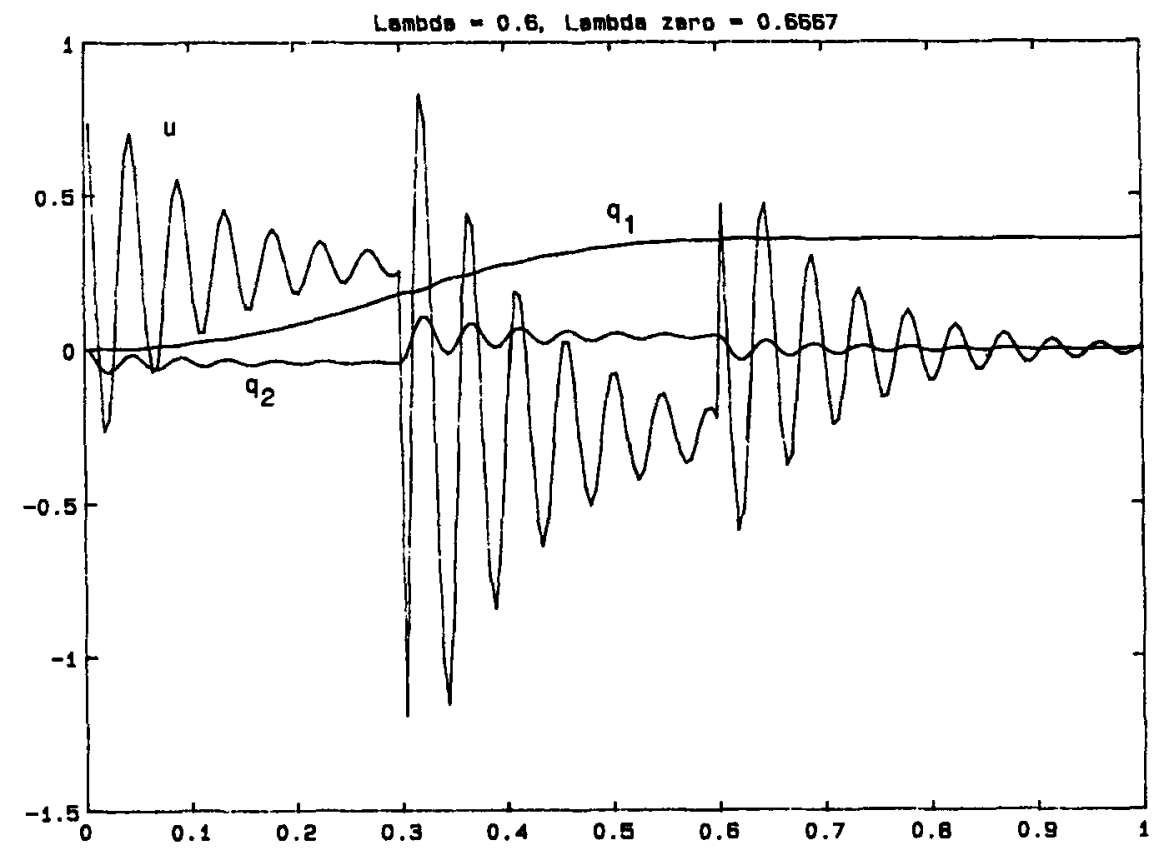

Figure 6(b). Torque, $q_{1}$ and $q_{2}\left(q_{2}\right.$ is multiplied by 5$)$. 


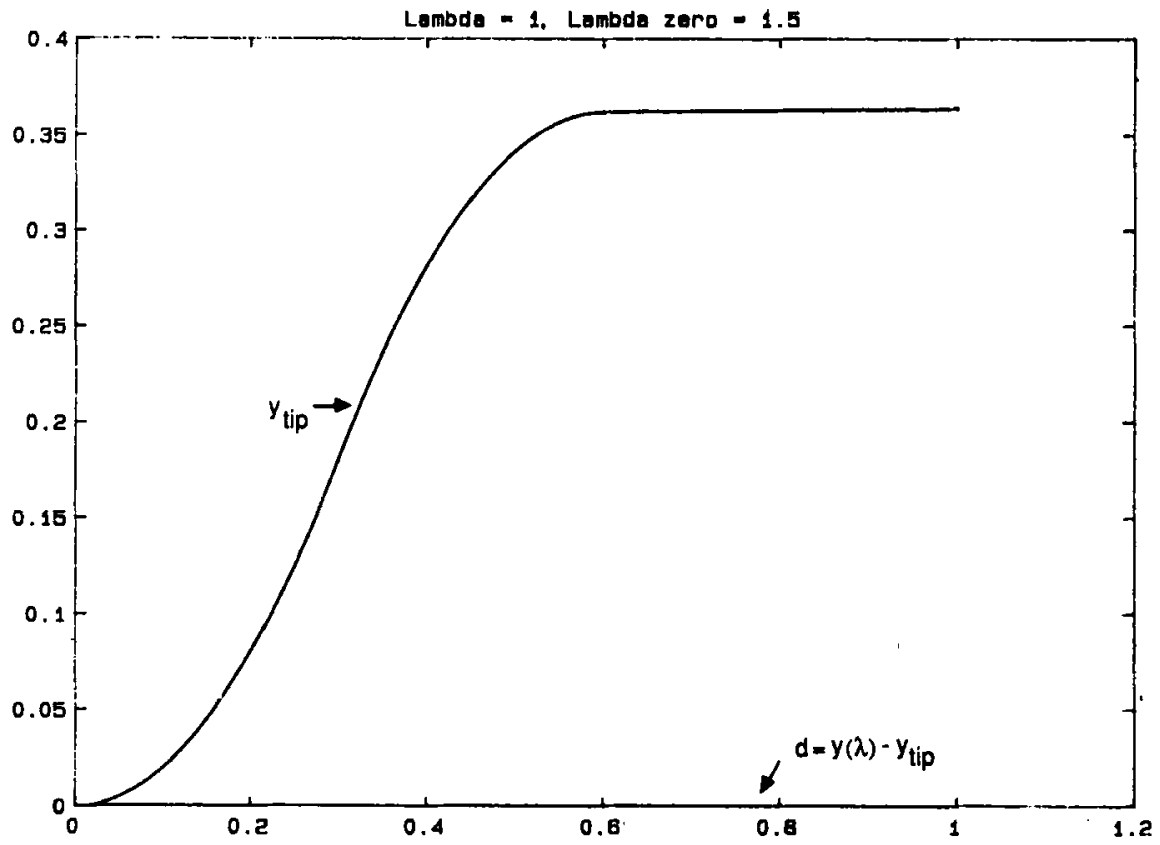

Figure 7(a). Outputs and their difference ( $d$ is multiplied by 5).

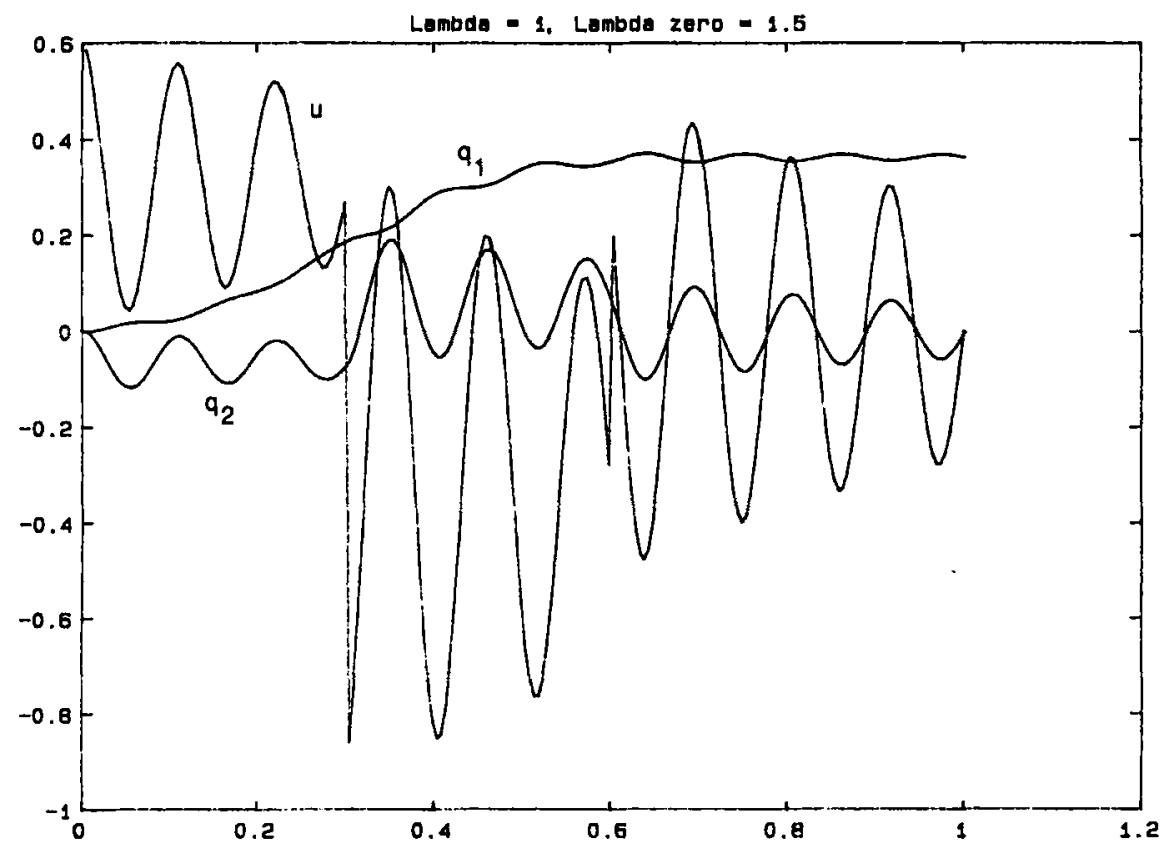

Figure 7(b). Torque, $q_{1}$ and $q_{2}$ ( $q_{2}$ is multiplied by 5 ). 


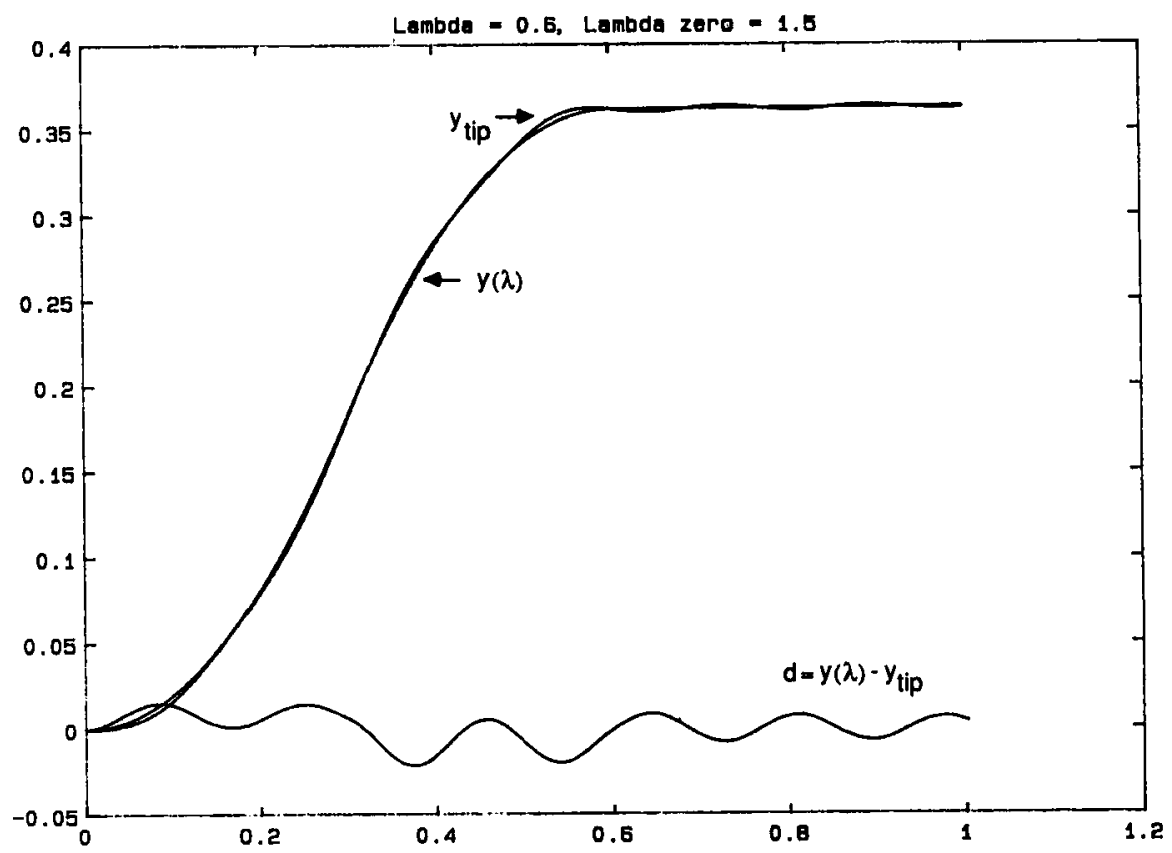

Figure 8(a). Outputs and their difference ( $d$ is multiplied by 5 ).

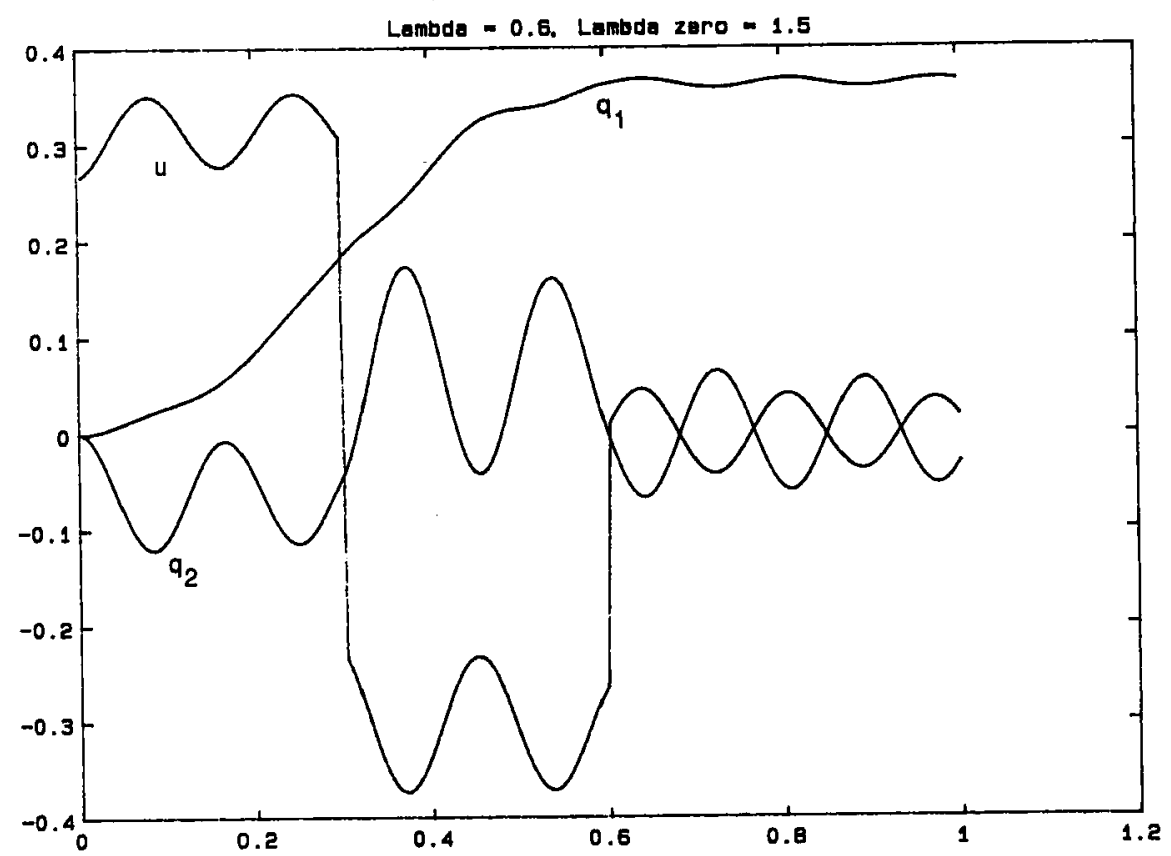

Figure 8(b). Torque, $q_{1}$ and $q_{2}\left(q_{2}\right.$ is multiplied by 5$)$. 
valuable reduction is obtained for the torque with still a good tracking performance of the tip.

\section{CONCLUSIONS}

The problem of exact reproduction of smooth trajectories for flexible robotic arms has been considered. A general framework has been presented for designing control laws based on system inversion. Open-loop reference torque generation and closed-loop state-feedback control, are always feasible when the unobservable dynamics associated to the chosen outputs is asymptotically stable. This is the critical issue to be addressed in the definition of the system outputs. In particular, the properties of the zero-dynamics--the nonlinear analogue of the concept of zeros of linear systems-play a central role.

It has been shown on a simple flexible arm that joint trajectories with bounded acceleration can be exactly reproduced in a inherently stable way, using the inversion-based control. This result can be generalized to flexible robot arms with any number of links, each of which has any finite number of flexible modes.

The same inversion strategy may become unstable if the control objective is to follow exactly a given end-effector trajectory. This can be seen from the instability of the associated zero-dynamics. The results available in the literature about the nonminimum phase characteristics of end-effector control using liner models of flexible arms $\mathrm{s}^{4.7}$ match with this observation. Noncolocation of actuators and outputs is usually a reasonable explanation of this effect.

However, it has been shown that other stable control strategies are feasible. In particular, there exists a continuous set of points along the flexible arm that can be assigned any desired smooth trajectory. This is the same as saying that the behavior of the flexible arm can be stiffened by feedback at any of these points. The simulation study confirms the intuitive idea that choosing as controlled output a point in this set which is closer to the end-effector results in a smaller tracking error of the tip, although in a larger torque requirement. Also, mechanical structures which are intrinsically stable from the point of view of end-effector trajectory tracking can be devised.

\section{References}

1. W. H. Sunada and S. Dubowsky, "On the dynamic analysis and behavior of industrial robotic manipulators with elastic members," Trans. ASME J. Dyn. Syst., Meas., Contr., 105, 42-51, 1983.

2. W. J. Book, "Recursive Lagrangian dynamics of flexible manipulator arms," Int. J. Robotics Res., 3, 87-101, 1984.

3. S. Nicosia, P. Tomei and A. Tornambe, Dynamic modeling of flexible manipulators, in 3rd IEEE Int. Conf. Robotics and Automation, San Francisco, 1986.

4. R. H. Cannon Jr. and E. Schmitz, "Initial experiments on the end-point control of a flexible one-link robot," Int. J. Robotics Res., 3, 62-75, 1984.

5. B. Siciliano and W. J. Book, "A singular perturbation approach to control of lightweight flexible manipulators", Int. J. Robotics Res., 7, 79-90, 1988. 
6. B. Siciliano, B. S. Yuan, and W. J. Book, "Model reference adaptive control of a one link flexible arm," in 25th IEEE Conf. Decision and Control, Athens, 1986.

7. C. Canudas de Wit and E. Van den Bossche, "Adaptive control of a flexible arm with explicit estimation of the payload mass and friction," in IFAC Int. Symp. on Theory of Robots, Vienna, 1986.

8. S. Nicosia, P. Tomei, and A. Tornambè, "Nonlinear controller and observer for a single-link flexible manipulator," in 4th IEEE Int. Conf. Robotics and Automation, Raleigh, 1987.

9. A. De Luca and B. Siciliano, "Joint-based control of a nonlinear model of a flexible arm," in American Control Conf., Atlanta, 1988.

10. A. K. Bejczy, "Robot Arm Dynamics and Control," Jet Propulsion Lab, California Inst. Technology, TM 33-669, 1974.

11. T. J. Tarn, A. K. Bejczy, A. Isidori, and Y. Chen, "Nonlinear feedback in robot arm control," in 23rd IEEE Conf. Decision and Control, Las Vegas, 1984.

12. S. N. Singh and A. A. Schy, "Robust torque control of an elastic robotic arm based on invertibility and feedback stabilization," in 24th IEEE Conf. Decision and Control, Ft. Lauderdale, 1985.

13. E. Bayo, "A finite-element approach to control the end-point motion of a single-link flexible robot," J. of Robotic Systems, 4, 63-75, 1987.

14. A. De Luca, P. Lucibello, and F. Nicolo, "Automatic symbolic modeling and nonlinear control of robots with flexible links," in IEEE Int. Workshop on Robot Control, Oxford, 1988.

15. R. M. Hirschorn, "Output tracking in multivariable nonlinear systems", IEEE Trans. Automatic Control, 26, 595-598, 1981.

16. R. M. Hirschorn, "Invertibility of multivariable nonlinear control systems," IEEE Trans. Automatic Control, 24, 855-865, 1979.

17. A. Isidori and C. Moog, "On the nonlinear equivalent of the notion of transmiss on zeros," in Modeling and Adaptive Control, C. I. Byrnes, K. H. Kurszanski Eds., Springer-Verlag, 1987.

18. A. De Luca, Dynamic control of robots with joint elasticity, in 5 th IEEE Int. Conf. Robotics and Automation, Philadelphia, 1988.

19. A. Isidori and C. I. Byrnes, "Local stabilization of minimum-phase nonlinear systems," Systems and Control Letters, 11, 9-17, 1988. 